\title{
Contraste de perspectivas y experiencias sobre compasión de enfermeras colombianas con 14 países
}

\section{Contrast of perspectives and experiences on compassion of Colombian nurses with 14 countries}

\author{
Lucero López-Díaz ${ }^{1}(\mathbb{D})$, Vilma Florisa Velasquez² ${ }^{(\mathbb{D})}$, Villerland Rodríguez $^{3}$ (D), Irena Papadopoulos ${ }^{4}$
}

Tipología: Artículo de investigación científica y tecnológica

Para citar este artículo: López-Díaz L, Velasquez V, Rodriguez V, Papadopoulos I. Contraste de perspectivas y experiencias sobre compasión de enfermeras colombianas con 14 países. Duazary. 2019 mayo; 16(2): 233-244. Doi: https://doi.org/10.21676/2389783X.2747

Recibido en abril 26 de 2018

Aceptado en diciembre 21 de 2018

Publicado en línea en marzo 15 de 2019

\section{RESUMEN}

Se exploraron puntos de vista y experiencias sobre compasión en la práctica de enfermeras colombianas que contrastan con los otros 14 países participantes del estudio internacional online. El estudio transversal exploratorio con survey internacional online con 10 preguntas (abiertas y cerradas) contó con 1323 enfermeras; 103 fueron de Colombia. Los datos cuantitativos colombianos fueron analizados en Excel y los cualitativos, en el programa Nvivo con análisis temático y consenso entre investigadores. La muestra colombiana contó con $83,5 \%$ docentes, y eligieron la definición de compasión como la "conciencia profunda de sufrimiento de los otros y el deseo de aliviar ese sufrimiento" (84,3\%). Piensan que la compasión puede ser enseñada $(64,1 \%)$ pero no es impartida $(45,1 \%)$ y es influenciada por la experiencia personal $(38,2 \%)$ y los valores culturales $(37,3 \%)$. Los relatos de Colombia revelan barreras sociopolíticas, organizacionales y educativas para la atención compasiva. Perciben compasión de sus pacientes $(84,7 \%)$, escasa compasión entre colegas (14,3\%) y casi nula compasión por parte de los gerentes (1\%). Sin embargo, emerge la naturaleza consciente e intencional de la compasión y el esfuerzo de enfermería por desarrollarla en cinco componentes: particularizar, invertir tiempo, estar presente, ir más allá y la defensa/abogacia. Se hacen evidentes algunas variaciones culturales de la compasión y formas de promoverla en un ejercicio profesional cada vez más transcultural.

Palabras clave: empatía; compasión; competencia cultural; enfermería transcultural.

1. Universidad Nacional de Colombia. Bogotá, Colombia. Correo: allopezdi@unal.edu.co - http://orcid.org/0000-0002-2157-763X

2. Universidad Nacional de Colombia. Bogotá, Colombia. Correo: vvelasquez@unal.edu.co - http://orcid.org/0000-0002-5232-9073

3. Universidad Nacional de Colombia. Bogotá, Colombia. Correo: vrodriguezgo@unal.edu.co - http://orcid.org/0000-0002-2157-763X

4. Middlesex University. London, United Kingdom. Correo: R.Papadopoulos@mdx.ac.uk- http://orcid.org/0000-0001-6291-4332 


\section{ABSTRACT}

Points of view and experiences about compassion were explored in the practice of Colombian nurses and a contrast was made with the other 14 participating countries of the international online study. The cross-sectional exploratory study with an online international survey with 10 questions (open and closed), had 1323 nurses, 103 were from Colombia. Colombian quantitative data were analyzed in Excel and qualitative data in Nvivo with thematic analysis and consensus among researchers. The Colombian sample counted with $83.5 \%$ teachers and they chose the definition of compassion as the "deep awareness of the suffering of others and the desire to alleviate that suffering" $(84.3 \%)$ in contrast to $59.5 \%$ of the online international study. Although, there are statistically significant differences between the countries of the international online study in the definition of compassion and some experiences, there are also similarities in several qualitative findings. The sociopolitical, organizational and educational barriers for compassionate care are evident. However, there is a conscious and intentional nature of the compassion and nursing effort to develop it. Some cultural variations of compassion and ways of promoting it in an increasingly transcultural professional exercise are evident.

Keywords: Empathy; Compassion; Cultural Competency; Transcultural Nursing.

\section{INTRODUCCIÓN}

a compasión es un ideal conductual de pro-

fesionalismo para quienes hacen parte de la atención en salud y está consagrada en algunas normas profesionales como valor en los sistemas de salud para la práctica profesional, derechos del paciente, estándar de calidad y guía de buena práctica $^{1-6}$.

La compasión ha sido definida como "una profunda consciencia del sufrimiento del otro y la voluntad de aliviarlo"7,8. En la literatura aparece como intercambiable con conceptos de simpatía o empatía pero se extiende porque busca una respuesta al sufrimiento observado y reconocido con reciprocidad e interdependencia, en muchos casos invisible $e^{1,5,8,9}$.

La importancia de la compasión es refrendada por pacientes y familias ${ }^{2,5}$. Informes de organismos de control, en algunos países, señalan una pobre atención en enfermería y sitúan la compasión para contrarrestarla ${ }^{8,10,11}$. No obstante, hay reacción a estas denuncias solicitando a los Gobiernos, gerentes y órganos rectores de prestación de servicios de salud considerar una visión menos centrada en los fundamentos del mercado (la eficiencia) y más en el paciente, considerando la complejidad de los sistemas de salud modernos; trascender la forma simplista de falta de compasión de los profesionales para abordar las barreras y facilitadores congruentes con la atención compasiva ${ }^{12,13}$.

El cuidado compasivo tiene efectos en los resultados en salud de los pacientes, en la recuperación y en el compromiso con el control de su salud; los pacientes se sienten escuchados y entendidos, lo que favorece la mejoría de los síntomas; es una herramienta eficaz para obtener información del paciente, para mejorar la atención y motivarlos ${ }^{5,6}$. Además, se ahorra en tiempo y costos; hay mayor satisfacción en el trabajo, bienestar individual y mejor salud mental $^{2,5,6}$. Sin embargo, la eficacia clínica, su viabilidad y las variaciones culturales requieren investigación adicional ${ }^{5}$.

El objetivo fue explorar puntos de vista y experiencias sobre compasión en la práctica de enfermeras colombianas y contrastar las semejanzas y diferencias con los demás países participantes (14) del estudio internacional online (EIO). 


\section{MATERIALES Y MÉTODOS}

\section{Tipo de estudio}

Estudio transversal exploratorio con encuesta en línea basado en la web con preguntas abiertas y cerradas. Participaron 15 países (Australia, Chipre - habla griega o turca-, Colombia, España, Estados Unidos, Filipinas, Grecia, Hungría, Israel, Italia, Noruega, Polonia, Reino Unido, República Checa y Turquía). Liderado por Papadopoulos con coinvestigadores voluntarios en cada país ${ }^{8,14}$.

La encuesta desarrollada por Irena Papadopoulos (IP) tiene 10 preguntas: tres abiertas y siete cerradas con reactivos de respuesta específico. La escala tiene validez de contenido y dado el carácter exploratorio del diseño no se calculó la confiabilidad. La traducción y retraducción al castellano fue ejecutada por un equipo español siguiendo los lineamientos propuestos para ello por la Organización Mundial de la Salud ${ }^{15}$.

\section{Muestra}

Se analiza la muestra colombiana que contó con 103 enfermeras de las 1323 del EIO. Fueron incluidas enfermeras de cualquier rol profesional y estudiantes de enfermería de último año.

\section{Análisis de datos}

Los datos cuantitativos de Colombia se analizaron en Excel, los resultados del estadístico Chicuadrado y de varianza se tomaron del EIO. Los datos cualitativos surgen de las preguntas abiertas: ¿Cómo definiría el término compasión? ¿Cómo se demuestra la compasión en la práctica asistencial? Escriba algunos ejemplos. En Colombia fueron compilados y categorizados con el programa de análisis cualitativo Nvivo ${ }^{\oplus}$, siguiendo las seis fases de análisis temático propuesto por Braun y Clarke ${ }^{16}$. Los hallazgos fueron el resultado del consenso y reflexividad del equipo colombiano durante seis sesiones (4 a 8 horas) y se hizo contraste con los temas de orden superior del $\mathrm{EIO}^{14}$.

\section{Declaración sobre aspectos éticos}

La encuesta garantizó el anonimato y fue respondida previo consentimiento informado. Se respetó la Declaración de Helsinki e igualmente fue considerada la Resolución 8430 de 1993 del Ministerio de Salud. Contó con aprobación del comité de ética (Middlesex University —-donde labora Papadopoluos, investigadora principaly la Universidad Nacional de Colombia).

\section{RESULTADOS}

Participaron 103 enfermeras colombianas (83,5\% docentes y 3,9\% asistenciales) y 3,9\% estudiantes de último año (3,9\%), 8,7\% no se identificaron. El 84,3\% define la compasión como la "conciencia profunda de sufrimiento de los otros y el deseo de aliviar ese sufrimiento".

La compasión en los relatos de Colombia tiene tanto de actitud, emoción o pensamiento con acciones de solidaridad y equidad: "Ser capaz de mirar al otro como un igual y acompañarlo con respeto en su tristeza en su alegría... Nunca una perspectiva moralista y sí una actitud de solidaridad" (Colombia-17). En la práctica es ofrecer cuidado "atendiendo al paciente no solo su parte biológica y su enfermedad. Verlo como ser humano, tener en cuenta sus creencias, sus sentimientos" (Colombia-69). Para algunos representa una connotación religiosa o cultural negativa relacionada con la lástima o de pena por el otro. Se equipara la compasión con humanización (10\%). 
En Colombia, la compasión es muy importante en enfermería (68,9\%), puede ser enseñada $(64,1 \%)$ pero no es impartida $(45,1 \%)$. Lo analizan así: "La compasión es uno de los sentimientos propios de la profesión... se adquiere con su práctica en el contacto con el otro/a. Es importante desde la enseñanza" (Colombia-23). "La compasión es un 'tema', 'asignatura' o 'tópico'... pienso que es necesario ayudar a reconocer... ¿Qué acciones compasivas reconozco que tengo hacia mí misma? ¿Con qué clase de personas soy más compasiva? ¿He recibido acciones compasivas de otros? ¿Cómo ha sido esa experiencia?” (Colombia-24). Mencionan algunas barreras en la enseñanza de la compasión: "Les enseñan más a ser máquinas y todo lo realizan porque toca" (Colombia-90). "Se ha enseñado que con el fin de ser objetiva... mostrar los sentimientos ha sido señal de debilidad... es casi como vivir anestesiado y al estar así es imposible percibir lo propio y lo de los otros" (Colombia-12). Se considera que la influencia más importante para el desarrollo de la compasión es la experiencia personal $(38,2 \%)$ y los valores culturales $(37,3 \%)$; piensan que los pacientes prefieren ser cuidados por enfermeras expertas con buenas habilidades interpersonales (83\%); valoran más el tratamiento médico $(47,4 \%)$ y la eficiencia que la compasión (42,3\%).

\section{Tema de orden superior l: estructura sociopolítica}

Son evidentes las barreras a la compasión por la contención de costos, la eficiencia, sobrecarga laboral y pocas garantías laborales:

"El principal obstáculo del cuidado compasivo en Colombia radica en las exigencias del modelo económico que pone en el centro no a las per- sonas sino el capital" (Colombia-26). "A las enfermeras asistenciales no se les reconoce ni en cargo, ni en salario sus estudios de posgrado. El salario no se compadece con sus responsabilidades... han abandonado el cuidado directo de los pacientes por administrar los recursos (facturar) debido a las normas existentes y a las exigencias institucionales" (Colombia-79).

Las participantes colombianas perciben compasión de sus pacientes $(84,7 \%)$, "son más compasivos los mismos pacientes... sienten pesar por las enfermeras que están sobrecargadas de trabajo, que se ven cansadas o que deben hacer tantas cosas al mismo tiempo, y prefieren no molestarlas" (Colombia-7).

En contraste, perciben escasa compasión entre colegas $(14,3 \%)$ y manifiestan: "Al profesional de enfermería no se le enseña la solidaridad con su colega, por tanto es muy difícil que se observe sentimientos de compasión entre colegas"(Colombia-5); y perciben casi nula compasión por parte de los gerentes (1\%) y se explica: "Considero que la compasión en el sistema de salud colombiano no se aplica ni por parte de las enfermeras, ni los pacientes, ni los gerentes; esto es el resultado de la implementación de un modelo que no considera importante la compasión y al cual le interesa más la productividad" (Colombia-81).

\section{Tema de orden superior II: naturaleza consciente e intencional de la compasión}

Se demuestra la compasión en la práctica asistencial con explicaciones, experiencias, percepciones donde es clara la naturaleza consciente e intencional de la compasión con similitudes a los componentes del $\mathrm{EIO}^{14}$ (Tabla 1). 
Tabla 1. Tema de orden superior II: Naturaleza consciente e intencional de la compasión: Componentes y acciones

\begin{tabular}{|c|c|}
\hline Componentes & Acciones \\
\hline \multirow{3}{*}{ Particularizar } & $\begin{array}{l}\text { Reconocer la singularidad de } \\
\text { sufrimiento }\end{array}$ \\
\hline & $\begin{array}{l}\text { Reconocer la singularidad } \\
\text { problemas de salud } \\
\text { (Enfermedad, vulnerabilidad, } \\
\text { orientación mental) }\end{array}$ \\
\hline & $\begin{array}{l}\text { Reconocer características } \\
\text { individuales } \\
\text { (Edad, género, cultura, } \\
\text { socioeconómicas) }\end{array}$ \\
\hline \multirow{4}{*}{ Invertir tiempo } & Ofrecer soporte \\
\hline & Escuchar \\
\hline & Desarrollar relación terapéutica \\
\hline & Educar \\
\hline \multirow{3}{*}{ Estar presente } & Acompañar \\
\hline & $\begin{array}{l}\text { Reconocer la importancia del } \\
\text { momento }\end{array}$ \\
\hline & Expresar afecto \\
\hline \multirow{6}{*}{ Ir más allá } & Entregarse \\
\hline & $\begin{array}{l}\text { compartir el sentido de lo } \\
\text { humano }\end{array}$ \\
\hline & $\begin{array}{l}\text { Involucrarse más allá de las } \\
\text { funciones }\end{array}$ \\
\hline & Involucrarse más allá del deber \\
\hline & Involucrarse con la familia \\
\hline & $\begin{array}{l}\text { Promover la competencia } \\
\text { cultural }\end{array}$ \\
\hline \multirow{3}{*}{$\begin{array}{l}\text { Defensa y } \\
\text { abogacía }\end{array}$} & Promover la igualdad \\
\hline & Desafiar la injusticia \\
\hline & Desafiar los estereotipos \\
\hline
\end{tabular}

Fuente: Adaptado del EIO ${ }^{14}$ con los datos del estudio colombiano.
Particularizar: reconoce la singularidad del sufrimiento, especialmente relacionado con el desplazamiento, vulnerabilidad, pobreza y desde allí se ven las características propias del curso de vida y la situación de salud: "En nuestro país las familias desplazadas sufren los efectos de la guerra, y aquí la enfermera es un soporte importante para aliviar este sufrimiento, estableciendo redes de apoyo, escuchando, realizando intervención en crisis... ante pérdidas materiales, accidentes naturales, violaciones, pobreza, discapacidad... es un ser muy cercano, capaz de aliviar el sufrimiento" (Colombia-16).

Invertir tiempo: se da importancia a invertir tiempo en la práctica compasiva y se hacen esfuerzos conscientes para superar las barreras a la compasión mediante acciones como: ofrecer soporte, escuchar, desarrollar la relación terapéutica y educar. Ofrecer soporte involucra confianza, solidaridad y acciones de ayuda en aspectos biológicos, psicológicos y sociales; aliviar el dolor, el sufrimiento, la incertidumbre, la tristeza; asesoría para la persona como para los familiares sobre la situación de salud, los procedimientos y expectativas; orientación (trámites). Escuchar implica una presencia plena con un contacto verbal o no verbal para entender y ofrecer soporte en la necesidad, más allá de lo visible, tanto para el paciente como para la familia; es una escucha activa abierta, con respeto "se mira a la persona, a la familia y se toma el tiempo para pensar en lo que le pasa, en sentir lo que están sintiendo... se dicen palabras dulces y alentadoras... se acompaña el discurso con una mirada y con el contacto físico... Se pone la persona por encima de las normas y las disposiciones, si es preciso hacerlo" (Colombia-55). 
Estar presente: tiene una estrecha relación con invertir tiempo, alta sensibilidad, presencia genuina y afectiva. Acompañar es un término común y aplica tanto a procesos de salud/ enfermedad, barreras para la atención, expresar afecto al momento que la persona está viviendo. Es común la frase: "Hay que ponerse en los zapatos de la persona a quien se cuida" (Colombia-78,95,98). En síntesis: "La compasión se demuestra cuando se toman acciones frente a las injusticias, las demoras, las malas prácticas. Cuando se es capaz de abogar por el otro en su situación de salud. Cuando se hacen las cosas bien con calidad y con interés. Cuando se antepone la necesidad del otro frente a otras cosas. Cuando estoy realmente presente ante el paciente y escucho sus necesidades y soy capaz de tomar parte de la solución" (Colombia-8).

Ir más allá: es entrega cuando hay reconocimiento del sufrimiento del otro y el compartir el sentido de lo humano: "Hacer con el otro lo que quisiéramos que hicieran con nosotros. Ponerse en el lugar del enfermo y su familia" (Colombia-79). Es involucrarse más allá de las funciones, del deber e involucrar la familia. "Cuando una enfermera realiza algo que no necesariamente está dentro de sus funciones con el fin de contribuir al bienestar del paciente, es decir, no es obligatorio llamar a un paciente que egresó de la clínica, pero una enfermera puede hacerlo... como ser humano no tanto como profesional, aunque la línea que divide eso puede ser difusa" (Colombia-66).

La competencia cultural aparece como reconocer la diversidad y evitar los juicios, los profesionales van más allá de las funciones, normas y hasta de su rol profesional. "No juzgar a los pacientes por sus preferencias sociales, religiosas, sexuales etc. que difieran de los que normalmente compartimos." (Colombia-89). "Aceptando las expresiones, las vivencias de los pacientes tal como son evitando juzgar y tratando de integrar estas experiencias en el cuidado del individuo" (Colombia-87). "Mostrar compasión es poner en ejercicio la autonomía de la enfermera aun cuando ello implica en ocasiones transgredir las normas y reglas institucionales o los propios valores. La compasión exige además competencias culturales" (Colombia-18).

Los relatos apuntan a adaptar acciones de cuidado y ajustar las rutinas laborales para atender la particularidad del sufrimiento y la diversidad de la persona cuidada y su familia: "Permito el ingreso de los familiares de las personas que están en la UCI a horas no establecidas; permitir a familiares acompañar a su familiar al final de la vida." (Colombia-57); "cuando brindo educación utilizo lenguaje sencillo, no utilizo tecnicismo que el paciente no entienda." (Colombia-82); "facilitando el empoderamiento de las personas, las familias y los grupos en el reconocimiento de sus formas de cuidado, la búsqueda de nuevas formas de cuidado y el uso crítico de los mismos" (Colombia-94).

Defensa y abogacía: reconoce las condiciones de vulnerabilidad, el sentido de solidaridad, la reivindicación de los derechos y defensa de la población cuidada como reconocimiento al conflicto político y demás barreras a la compasión. Es asumir riesgos: "En una E.S.E. de Bogotá al servicio de urgencias llegó un niño indígena Embera-Katio sin registro civil, hecho que en Colombia se entiende como maltrato infantil por negligencia... Ante la angustia de sus padres... [me] encontré con que no sabían qué era el registro civil, ni le veían importancia alguna pues ellos no se concebían como colombianos sino como Emberas y por tanto no tenía sentido tener documento que los identificara como colombianos. Ante la situación decidí sugerir a los padres de familia el cumplimiento de este requisito y no hacer el reporte. Actué contra la norma y aún en contra de mi valor de 
veracidad, pero con el convencimiento de que por encima de la norma están las personas y sus realidades particulares" (Colombia-18).

Hay acciones para promover la igualdad y desafiar prejuicios o estereotipos bajo la consideración de compartir la esencia de ser humanos. "Ayudando a aliviar el dolor y mostrarle al usuario (por ejemplo, un violador, ladrón, pedófilo) que él es una persona... Ayudar a un usuario así no sea de mi religión, así vaya en contra de mis principios (esto es semejante a neutralidad) ... pero ante un dolor y una ofensa es compasión" (Colombia-75).

\section{DISCUSIÓN}

En el estudio colombiano predominan las enfermeras docentes $(83,5 \%)$ mientras en el $\mathrm{EIO}^{8,14}$ predominaron las asistenciales $(45,4 \%)$, y solo el 29,3\% eran docentes. Así, los resultados colombianos son permeados por la visión de enfermeras docentes. La compasión entendida en Colombia $(84,3 \%)$ como la "conciencia profunda de sufrimiento de los otros y el deseo de aliviar ese sufrimiento" es compartida por el grupo de Israel y España. En Chipre, Filipinas y Reino Unido se definió como "empatía y amabilidad". El análisis de varianza (ANOVA) del EIO ${ }^{8}$ mostró diferencias significativas en la definición de compasión entre los países n; $\mathrm{F}(14,1106)=$ $21,035, \mathrm{P}<0,0001$.

El término compasión puede traer valores, normas y prácticas culturalmente específicas ${ }^{7}$. Para algunas colombianas tiene una connotación negativa relacionada con aspectos religiosos de lástima, de piedad y en sus narrativas intercambian el término por el de humanización. Otros estudios han encontrado la lástima/piedad (en inglés pity) como sinónimo de compasión pero han puntualizado la diferencia con la compasión porque en la compasión la persona es vista como alguien digno para ser ayudado ${ }^{4,6,13}$ La reciente revisión sistemática describe dos condiciones de la compasión: la presencia del sufrimiento de una persona y la de una persona para aliviarla ${ }^{5}$. Por tanto, la compasión implica reconocer, responder, comprender y tratar activamente el sufrimiento del otro como se hace evidente en este estudio y el $\mathrm{EIO}^{14}$. La naturaleza dinámica y temporal de la compasión se protege de un enfoque prescriptivo para volverse en una combinación de habilidades, virtudes de los cuidadores, intuición, afecto y la presencia, por lo que puede ser titulada y adaptada con el tiempo, aunque requiere más investigación clínica ${ }^{5}$.

A pesar de las diversas estructuras políticas y de los sistemas de salud en los 15 países participantes del $\mathrm{EIO}^{14}$, fueron evidentes los efectos de las barreras organizativas (limitaciones de tiempo, sobrecarga laboral, escasez de personal) y de estructuras sociopolíticas (modelo de negocio o línea de producción) sobre el ejercicio de la compasión. Esa cultura organizacional de rapidez y priorización en la eficiencia termina perjudicando la calidad del cuidado y la posibilidad de establecer una atención compasiva ${ }^{5,8,14,17-19}$.

Aunque el contexto es adverso para la compasión en los 15 países, en Colombia se agrava con la escasa compasión entre colegas $(14,3 \%)$ contrario a otros países del $\mathrm{EIO}^{8}$ como Australia, Chipre (habla griega), Estados Unidos, España, Grecia, Hungría, Israel, Reino Unido y Noruega, lugares donde hay mayor se percibe entre $66 \%$ y $48,1 \%$ de compasión entre colegas. Además, no son percibidas muestras de compasión de los gerentes en Australia, Italia e Israel, en Colombia solo $1 \%$, el $\mathrm{EIO}^{8}$ es de $4,3 \%$. Sin embargo, esto mejora en Filipinas $(39,6 \%)$ y Chipre $(20,8 \%)$, quizá está asociado a valores sociales colectivos (Filipinas, Chipre, Turquía) y al mismo tiempo es contradictorio porque otros países con valores similares no perciben compasión de los gerentes (Australia, Israel, Italia). Por consiguiente, factores como la eficiencia, productividad $y$ 
miedo del líder o gerente a ser percibido como poco profesional pueden influir en la expresión de compasión a sus empleados. Igualmente, se afirma el papel trascendental de los líderes/ gerentes para fomentar la cultura de la compasión en las organizaciones.

Las enfermeras colombianas perciben mayor compasión de los pacientes $(84,7 \%)$, tanto como las colaboradoras en Italia $(84,6 \%)$, Filipinas (51\%), Turquía $(55,4 \%)$ y Polonia $(55,7 \%)$ del $\mathrm{EIO}^{8}$ indicando un proceso relacional de intercambio y ayuda ${ }^{20}$. Aunque se tiende a priorizar las necesidades de los pacientes, los profesionales de la salud necesitan de entornos seguros y compasivos, es necesario explorar cómo se puede lograr la compasión para el personal, los pacientes y las familias ${ }^{9}$. Si líderes o gerentes toman la compasión como un comportamiento aceptable y lo promueven en el trabajo tanto enfermeras como los demás integrantes de la organización, serán más propensos a adoptar tal comportamiento de compasión con sus colegas y pacientes ${ }^{8}$. Es necesario comprender que el desarrollo de la compasión está enmarcado en un sistema complejo de atención en salud donde las personas requieren el apoyo de los servicios sociales y otras esferas de la sociedad, por tanto, la presión no debería estar solamente en las enfermeras ${ }^{14}$. Las barreras descritas pueden contribuir a un alto nivel de estrés en el personal y al conflicto entre los ideales profesionales. El proceso de compasión debe trascender el interés profesional o la experiencia individual y enfatizar en la organización para crear y promover una cultura de atención compasiva ${ }^{9}$.

Hay debate sobre la compasión como innata, aprendida o la mezcla de las dos ${ }^{8,11,21}$. Aunque en Colombia se estima que puede ser enseñada para el EIO ${ }^{8}$ se presentan diferencias significativas entre países. Esto puede indicar dos asuntos críticos: la posibilidad de adquirir habilidades para la atención compasiva con el diseño de métodos apropiados de entrenamiento; y cómo desarrollar y sostener la compasión en una organización multicultural.

Quizá un enfoque individualizado, experiencial, de modelamiento de rol y de una práctica reflexiva (en la propia vida y el quehacer profesional) para el entrenamiento y la atención compasiva puede mitigar un enfoque prescriptivo. Se valora el aprender de los propios pensamientos y emociones, pero requiere de apoyo y entrenamiento. Este enfoque posibilitaría el desarrollo de atención compasiva adaptada tanto para los profesionales como para los pacientes ${ }^{4,5,9,22}$.

Procesos de entrenamiento en atención plena (mindfulness) y compasión han mostrado beneficios a nivel clínico, mostrando otros caminos investigativos y de entrenamiento de la compasión $^{23}$. El estudio corrobora desafíos sociopolíticos, organizacionales y educativos para lograr atención con compasión.

El estudio permite reconocer variaciones culturales de la compasión ${ }^{5,18}$. Algunas colaboradoras de Colombia prefieren el término humanización frente al de compasión probablemente porque en el entorno suramericano es común su utilización ${ }^{24}$; indican un imperativo para mejorar el contacto humano y la dignidad de las personas en los servicios de salud, y pasa a ser parte de las políticas en salud; sin embargo, dadas las dimensiones de la compasión, no deberían proponerse como intercambiables. Tanto en Colombia como en el EIO ${ }^{14}$ las explicaciones, experiencias y percepciones permiten encontrar el carácter consciente e intencional de la compasión compartido en los cinco componentes (Tabla 1); sin embargo, la secuencia de los componentes y algunas acciones tienen particularidades en Colombia.

El grupo de Colombia demuestra la capacidad intencional de acercarse al sufrimiento del 
otro, prestar atención a ese sufrimiento y entrar en sintonía con él, y sin juicios poder moverse para buscar su alivio o prevención generando un razonamiento, un comportamiento, una emoción ${ }^{17,25}$. Logra cierta competencia cultural al adaptar o promover acciones de cuidado que en ocasiones subvierten la normatividad institucional o del sistema y las propias funciones. Aparece como propio del contexto colombiano la solidaridad frente al desplazamiento interno, desigualdad e inequidad.

En el $\mathrm{EIO}^{14}$ aparece el componente personalizar, pero en Colombia se denominó particularizar, al final es entendido como sinónimo de individualizar o personalizar. Las enfermeras logran considerar las características individuales del paciente, sus particularidades en salud y la expresión singular del sufrimiento. El tener tiempo es ofrecer una atención particularizada, integral y con presencia. Este componente aparece en diversos estudios como "práctica de la compasión". Esto implica acciones como hacer algo por el otro; pequeñas cosas, pequeños actos de bondad; ir más allá; acciones guiadas por la intuición como mantener el silencio, escucha atenta, la postura y el tono de voz; mostrar comprensión; dejar descansar al paciente y trascender el enfoque en la tarea $a^{5,10,12,20,25}$.

Estar presente tiene interrelación con la calidad del tiempo ofrecido a los pacientes, algo común para el grupo de colaboradoras colombianas y del $\mathrm{EIO}^{8,14}$. La presencia implica la construcción de un espacio intencional de expresión de sentimientos y preferencias; de un contacto que puede moverse de "estar ahí" a "estar con" a "relacionarse con"; una conexión ilustrada con la metáfora "ponerse en los zapatos del otro" encontrada en este estudio y de peso significativo en la comprensión de la atención compasiva por parte de los pacientes trae un impacto positivo en el bienestar general, aunque es un desafío

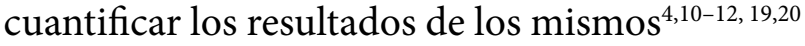

"Ir más allá" es una expresión del coraje, fuerza e intencionalidad de las enfermeras por responder a la unicidad de las personas, por sobrepasar las barreras y afrontar con valor lo que consideran moralmente correcto y elegir el curso de una acción hacia una práctica compasiva que lleva en ocasiones a un riesgo del bienestar personal de las enfermeras,111. El ir más allá tiene un elemento del coraje moral que lleva a la enfermera a superar los obstáculos con fuerza y sabiduría en condiciones difíciles y en conflictos abrumadores.

Les lleva a entender e interpretar las situaciones personales y clínicas y en consecuencia a abogar por los pacientes como un elemento de la práctica compasiva ${ }^{8,27}$

La compasión guía juicios y acciones en el ámbito de los derechos, las libertades y la diversidad, cumpliendo una función en la cooperación y la protección de los más débiles sugestiva de un juicio de equidad y justicia ${ }^{7,14}$. El estudio puede dar claves sobre el aspecto sociológico de compasión que muestra la interacción entre coerción, compasión, procesos interpersonales e institucionales ${ }^{20}$.

En general, las acciones de los componentes tanto de este estudio como del $\mathrm{EIO}^{14}$ son visibles e invisibles y difíciles de cuantificar con probable escaso valor para quienes desarrollan o gestionan los servicios y generalmente son advertidos por su ausencia. Pacientes, familiares o comunidad son considerados con sus necesidades como seres únicos como eje de una atención culturalmente competente y compasiva ${ }^{28,29}$. Sin embargo, la invisibilidad de las acciones de compasión puede tener implicaciones significativas para su reconocimiento, promoción y evaluación en la práctica ${ }^{9,20}$. 
Finalmente, el contraste de los datos cuantitativos y cualitativos del estudio colombiano con los de los 14 países participantes del $\mathrm{EIO}^{8,14}$ hacen este material relevante para mostrar las variaciones culturales de la compasión en salud y permite mostrar cómo el entorno cultural y las experiencias influyen en la forma de definir y expresar la compasión. Se necesitan más investigaciones para explorar diferencias culturales en la promulgación de la compasión en una enfermería de gran movilidad por el mundo. Conviene incluir entrevistas en profundidad, grupos focales y estudios de casos en tres niveles: la compasión en la estructura organizativa; la compasión entre colegas; la compasión expresada y comprendida por los pacientes y la educación para la compasión a los profesionales en formación y los que ejercen.

\section{LIMITACIONES DEL ESTUDIO}

La muestra en Colombia puede tener sesgos de selección (mayoría docentes), al utilizar prioritariamente las redes académicas de la primera investigadora. En el survey online no se registraron pormenores demográficos limitando el análisis frente a la diversidad en el país. Aunque se buscó superar la limitación de utilizar literatura anglo céntrica del $\mathrm{EIO}^{14}$ con la base LILACS de 119 referencias, solo tres artículos se pudieron incorporar. Desde Google Académico (español y portugués) se lograron identificar ocho documentos de los cuales tres son tesis de maestría de Portugal.

\section{DECLARACIÓN SOBRE CONFLICTO DE INTERESES}

Los autores declaran no tener ningún conflicto de interés. Existió apoyo interno de las instituciones universitarias para el desarrollo del proyecto.

\section{REFERENCIAS BIBLIOGRÁFICAS}

1. Burridge LH, Winch S, Kay M, Henderson A. Building compassion literacy: Enabling care in primary health care nursing. Collegian [Internet]. 2015; Disponible en: http://www.collegianjournal.com/article/ S1322-7696(15)00081-5/pdf

2. Papadopoulos I, Ali S. Measuring compassion in nurses and other healthcare professionals: An integrative review. Nurse Educ Pract [Internet]. 2016;16(1): 133-9. Disponible en:http:// www.sciencedirect.com/science/article/pii/ S1471595315001298?_rdoc=1\&_fmt=high\&_ origin=gateway\&_docanchor $=\& \mathrm{md} 5=\mathrm{b} 8429449$ ccfc9c30159a5f9aeaa92ffb

3. Barker R, Cornwell J, Gishen F. Introducing compassion into the education of health care professionals; can Schwartz Rounds help? J Compassionate Health Care; [Internet]. 2016; 3(1): 3. Disponible en: https://doi.org/10.1186/ s40639-016-0020-0

4. Sinclair S, McClement S, Raffin-Bouchal S, Hack TF, Hagen NA, McConnell S, et al. Compassion in Health Care: An Empirical Model. J Pain Symptom Manage [Internet]. 2016; 51(2): 193203. Disponible en: https://doi.org/10.1016/j. jpainsymman.2015.10.009

5. Sinclair S, Norris JM, Mcconnell SJ, Chochinov HM, Hack TF, Hagen N a, et al. Compassion: a scoping review of the healthcare literature. BMC Palliat Care [Internet]. 2016; (January): 1-16. Disponible en: https://doi.org/10.1186/ s12904-016-0080-0

6. Strauss C, Taylor BL, Gu J, Kuyken W, Baer R, Jones $\mathrm{F}$, et al. What is Compassion and How Can We Measure it? A Review of Definitions and Measures. Clin Psychol Rev [Internet]. 2016; 47: 15-27. Disponible en: https://doi.org/10.1016/j. cpr.2016.05.004 
7. Goetz JJL, Keltner D, Simon-Thomas E. Compassion: an evolutionary analysis and empirical review. Psychol Bull [Internet]. 2010; 136(3): 351-74. Disponible en: https://doi. org/10.1037/a0018807

8. Papadopoulos I, Zorba A, Koulouglioti C, Ali S, Aagard M, Akman O, et al. International study on nurses' views and experiences of compassion. Int Nurs Rev [Internet]. 2016; 63(3): 395-40. Disponible en: https://doi.org/10.1111/inr.12298

9. Dewar B. Cultivating compassionate care. Nurs Stand [Internet]. 2013; 27(34): 48-55. Disponible en: https://www.researchgate.net/profile/Maxine_ Pryce-Miller/publication/266973445_Developing_ compassion_in_pre-registration_education/ links/5534a44c0cf2f2a588b26277.pdf

10. Bramley L, Matiti M. How does it really feel to be in my shoes? Patients' experiences of compassion within nursing care and their perceptions of developing compassionate nurses. J Clin Nurs [Internet]. 2014; 23(19-20): 2790-9. Disponible en: https://doi.org/10.1111/jocn.12537

11. Curtis K. Learning the requirements for compassionate practice: student vulnerability and courage. Nurs Ethics [Internet]. 2014; 21(2): 210-23. Disponible en: https://doi. org/10.1177/0969733013478307

12. Christiansen A, O'Brien M, Kirton J, Zubairu K, Bray L. Delivering compassionate care: The enablers and barriers. Br J Nurs [Internet]. 2015; 24(16): 833-7. Disponible en: https://doi. org/10.12968/bjon.2015.24.16.833

13. Crawford P, Brown B, Kvangarsnes M, Gilbert P. The design of compassionate care. J Clin Nurs [Internet]. 2014; 23(23-24): 3589-99. Disponible en: https://doi.org/10.1111/jocn.12632

14. Papadopoulos I, Taylor G, Ali S, Aagard M, Akman O, Alpers L-M, et al. Exploring Nurses Meaning and Experiences of Compassion: An International Online Survey Involving 15
Countries. J Transcult Nurs [Internet]. 2017; 28(3): 286-95. Disponible en: https://doi. org/10.1177/1043659615624740

15. World Health Organization. Process of translation and adaptation of instruments. 2014. Disponible en: https://www.who.int/ substance_abuse/research_tools/translation/en/

16. Braun V, Clarke V. Using thematic analysis in psychology. Qual Res Psychol [Internet]. 2006; 3(2): 77-101. Disponible en: http://eprints.uwe. ac.uk/11735/

17. Menage D, Bailey E, Lees S, Coad J. A concept analysis of compassionate midwifery.

18. J Adv Nurs. 2017; 73(3): 558. DOI: 10.1111/ jan. 13214

19. Singh P, King-Shier K, Sinclair S. The colours and contours of compassion: A systematic review of the perspectives of compassion among ethnically diverse patients and healthcare providers. PLoS ONE [Internet]. 2018; 13(5): 1-18. Disponible en: https://doi.org/10.1371/journal.pone.0197261

20. Reis Silva AM. A compaixão entre enfermeiros em saúdeinfantilepediatria: análise do conceito [Tesis em internet]. Escola Superior de Enfermagem do Porto; 2014. Disponible en: https:// comum.rcaap.pt/bitstream/10400.26/9491/1/ D is s e r t a \% C $3 \%$ A 7 \% C $3 \%$ A 3 o \% 20 Enf\%C2\%AA\%20Ana\%20Reis.pdf

21. Brown B, Crawford P, Gilbert P, Gilbert J, Gale C. Practical compassions: repertoires of practice and compassion talk in acute mental healthcare. Sociol Health Illn [Internet]. 2014; 36(3): 383-99. Disponible en: https://doi. org/10.1111/1467-9566.12065

22. Curtis K. 21st Century challenges faced by nursing faculty in educating for compassionate practice: Embodied interpretation of phenomenological data. Nurse Educ Today [Internet]. 2013; 33(7): 46-50. Disponible en: https://doi.org/10.1016/j. nedt.2013.05.007 
23. Richardson C, Percy M, Hughes J. Nursing therapeutics: Teaching student nurses care, compassion and empathy. Nurse Educ Today [Internet]. 2015; 35(5): e1-e5. Disponible en: https://doi.org/10.1016/j.nedt.2015.01.016

24. Dunne S, Sheffield D, Chilcot J. Brief report: Selfcompassion, physical health and the mediating role of health-promoting behaviours. J Health Psychol [Internet]. 2016;28(7): 993-9. Disponible en: https://doi.org/10.1177/1359105316643377

25. Zambrano MLC. La humanización de la atención en los servicios de salud: un asunto de cuidado. Rev Cuid [Internet]. 2016; 7(1): 122731. Disponible en: https://doi.org/10.15649/ cuidarte.v7i1.300

26. Nunes Coelho CS. A compaixão dos enfermeiros perante a criança esuafamília, em cuidados paliativos [Tesis em internet]. Escola Superior de Enfermagem do Porto; 2015. Disponible en:https://comum. rcaap.pt/bitstream/10400.26/9759/1/Claudia\%20 Nunes_\%20Disserta\%C3\%A7\%C3\%A3o.pdf
27. Cameron RA, Mazer BL, Deluca JM, Mohile SG, Epstein RM. In search of compassion: A new taxonomy of compassionate physician behaviours. Health Expect [Internet]. 2015; 18(5): 1672-85. Disponible en: https://doi. org/10.1111/hex.12160

28. Sadooghiasl A, Parvizy S, Ebadi A. Concept analysis of moral courage in nursing: A hybrid model. Nurs Ethic. 2016; 25(1): 6-19. Disponible en: https://doi.org/10.1177/0969733016638146

29. Kvangarsnes M. Nurses' Perspectives on Compassionate Care for Patients with Exacerbated Chronic Obstructive Pulmonary Disease. J Allergy Ther [Internet]. 2013; 4: 158.

30. Petroulias P, Groesbeck L, Wilson FL. Providing Culturally Competent Care in Home Infusion Nursing. J Infus Nurs [Internet]. 2013; 36(2): 108-14. 